\title{
Uptake of HIV screening in genitourinary medicine after change to "opt-out" consent
}

\author{
Belinda Stanley, Jane Fraser, N H Cox
}

Department of Genitourinary

Medicine, North

Cumbria Acute

Hospitals Trust,

Cumberland

Infirmary, Carlisle

CA2 7HY

Belinda Stanley

consultant

Jane Fraser

senior clinical nurse

specialist

Department of

Dermatology, North

Cumbria Acute

Hospitals Trust

N H Cox

consultant

Correspondence to:

B Stanley

Belinda.Stanley@

ncumbria-acute.

nhs.uk

BMJ 2003;326:1174
"Routine" serological testing of patients in genitourinary medicine clinics screens for syphilis but not HIV, hepatitis B, or hepatitis C. Viral hepatitis and HIV are transmitted by sexual intercourse. National anonymised data from routine serological tests showed that patients in genitourinary medicine have a higher risk of testing positive for antibodies to HIV than does the general population. ${ }^{1}$ The national sexual health strategy states that $40 \%$ of new patients attending genitourinary medicine clinics should be screened for HIV infection by 2004 and $60 \%$ by $2007 .{ }^{2}$ Anticipating these targets, we audited our testing rates in our clinic and introduced change to increase uptake.

\section{Participants, methods, and results}

We examined case notes for 200 consecutive new patients attending genitourinary medicine clinics before August 2001 (100 at each clinic site of the genitourinary medicine department in North Cumbria) for blood tests requested (syphilis, HIV, hepatitis $\mathrm{B}$, hepatitis $\mathrm{C}$ ) and reasons given by the patients for declining screening. Because the rate of HIV testing was less than $60 \%$, opt-out screening was introduced to encourage uptake.

Subsequent patients were given a leaflet from their clinic explaining the tests recommended and offered routinely as part of the screening for sexually transmissible infection. This information was repeated by the doctor during the consultation. Patients were specifically asked whether they wanted to have all the tests offered, and any concerns were discussed. All patients spoke English. Pre-test counselling provided by health advisers was offered but not mandatory.

We audited patients' notes from 200 more consecutive new patients. Changing to opt-out HIV screening significantly increased uptake from 35\% to $65 \%$ but did not affect the uptake of syphilis screening (table). All patients who were tested for HIV also chose to be tested for syphilis. A quarter of patients, however, chose not to have blood taken. Some patients deferred being tested until the end of a three-month "window" period. We did not identify any new cases of HIV infection or syphilis. We did, however, identify two new cases of hepatitis C, both in former intravenous drug users who had not previously acknowledged this risk. No patient expressed dissatisfaction with the screening policy.

\section{Comment}

Introducing "routine" serological testing increased the acceptability and uptake of HIV screening. This is in line with the recommendations of the national strategy for sexual health and HIV. ${ }^{2}$

Some patients attending genitourinary medicine clinics assume that testing for HIV infection is routine. Patients who are recognised to have a higher risk for bloodborne viruses may be offered screening for
Number (percentage; 95\% confidence intervals) of consecutive new patients attending genitourinary medicine clinics tested for syphilis and HIV infection before and after the introduction of "opt-out" screening in 2001

\begin{tabular}{lcc} 
Period & $\begin{array}{c}\text { Tested for syphilis } \\
(\mathbf{n = 2 0 0 )}\end{array}$ & $\begin{array}{c}\text { Tested for HIV } \\
(\mathbf{n}=\mathbf{2 0 0})\end{array}$ \\
\hline Before Aug 2001 & $154(77 ; 71$ to 83$)$ & $70(35 ; 28$ to 42$)$ \\
\hline After Sept 2001 & $148(74 ; 67$ to 80$)$ & $130(65 ; 71 \text { to } 83)^{\star}$ \\
\hline
\end{tabular}

${ }^{*} \mathrm{P}<0.001, \chi^{2}$ test.

hepatitis and HIV. This strategy of offering targeted serological screening after the recognition of risk (optin), rather than as part of routine screening (opt-out), requires that the risk be recognised by staff, and this may result in a notable proportion of infections being missed. ${ }^{3}$ In a recent study of genitourinary medicine consultants, two fifths believed that the proportion of patients tested for HIV in their clinics was too low, even though doctors overestimated the testing rates. "Routine" antenatal screening for HIV infection has been well accepted in our local community $(96 \%$ of pregnant women screened for rubella were also screened for HIV). We believe that patients attending genitourinary medicine clinics should not receive a service that, by comparison, is suboptimal.

The concern that introducing "routine" HIV screening for new genitourinary medicine patients might reduce the uptake of syphilis screening was not supported by our data. The low local prevalence of HIV infection may have encouraged the uptake of screening in antenatal and in genitourinary medicine clinics, but recent legal judgments in Scotland could have been a dissuasion. ${ }^{5}$

It would be unwise to extrapolate the results of a study from a rural area and to expect similar outcomes in urban areas with higher prevalence of HIV, but introducing "routine" screening for HIV and hepatitis in genitourinary medicine was clearly acceptable and significantly increased uptake.

Contributors: BS conceived and designed the study, helped to analyse and interpret the data, drafted and revised the article, and shared in the final approval. JF helped to analyse and interpret the data, and shared in the final approval. NHC advised on writing, submitting, and revising the article, and shared in the final approval. BS is the guarantor.

Funding: None.

Competing interests: None declared.

1 Unlinked Anonymous Surveys Steering Group. Prevalence of HIV and hepatitis infections in the United Kingdom 2000. London: Department of Health, 2001.

2 The National Strategy for Sexual Health and HIV. Department of Health, London, 2001. www.doh.gov.uk/nshs/strategyhtm (accessed 12 march 2003)

3 Catchpole MA, Mercey DE, Nicoll A, Rogers PA, Simms I, Newham J, et al. Continuing transmission of sexually transmitted diseases among patients infected with HIV-1 attending genitourinary medicine clinics in England and Wales. BMJ 1996;312:539-42.

4 British Co-operative Clinical Group. Screening for HIV infection in genitourinary medicine clinics: a lost opportunity? Sex Transm Infect 2000;76:307-10.

Bird SM, Leigh Brown AJ. Criminalisation of HIV transmission: implications for public health in Scotland. BMJ 2001;323:1174-7.

(Accepted 17 January 2003) 